\title{
Covariation among elements of rattlesnake posture: Potential interspecific signals
}

\author{
FRANK IGLEHART and DAVID CHISZAR \\ University of Colorado, Boulder, Colorado 80309
}

\begin{abstract}
Two rattlesnakes were photographed in various behavioral states from two perspectives (from above and from the side) simultaneously, and 26 measures of posture were taken from each pair of pictures. A total of $\mathbf{4 8}$ pairs of pictures were digitized in this manner. These measures were then subjected to a correlational analysis. Various measures taken from the side view were significantly correlated with each other and with measures taken from the top view. For example, the degree to which the head and neck were elevated above the floor (as seen from side view) was correlated with the number of S-shaped loops in the body (as seen from top view). Both sets of measures were, in turn, associated with the snakes' momentary state of defensiveness. Hence, a bird viewing the snake from above could diagnose the snake's behavioral state by cues which are quite different from those which could be used for the same purpose by rodents or canids that would view the snake from a horizontal perspective. Although we have not established that these cues are in fact being utilized by birds, rodents, or canids (or any other animal), the fact remains that covarying postural elements are associated with particular behavioral states and that they may be useful cues for prey or predators.
\end{abstract}

The purpose of this study was to determine which (if any) elements of rattlesnake posture vary systematically across different stimulus conditions which normally produce distinctly different behavioral states, for example, rest, alertness, exploration, predation, and defense. Such data can be used to develop hypotheses about potential interspecific signal value of particular postural elements. Hence, this project examines the potential role of the rattlesnake as a source of visual information which might be used by prey and/or predators. Visual information arising from rattlesnake postures has not heretofore been subjected to detailed analysis, probably because the dramatic auditory cues deriving from the rattle have preempted the attention of investigators (Klauber, 1972).

\section{METHOD}

\section{Subjects and Maintenance Conditions}

Two rattlesnakes were used in this study: a pygmy rattlesnake, Sistrurus miliarus (about $30 \mathrm{~cm}$ long, 3 years old), and a western diamondback, Crotalus atrox (about $107 \mathrm{~cm}$ long, 5 years old). The first specimen was in the collection of the Baltimore City Zoo, Baltimore, Maryland, and was observed in a plywood cage $(45 \times 45 \times 40 \mathrm{~cm})$ with screeen top and glass front. The second snake was in the collection of the second author and was observed in a glass cage $(90 \times 30 \times 45 \mathrm{~cm})$. Both animals had been in captivity more than a year, had been accepting rodent prey readily, and had been living in cages similar to the observation units described above. Transfer to the observation cages occurred just prior to photographic recording. Temperature was $25^{\circ} \mathrm{C} \pm 2^{\circ} \mathrm{C}$ for both snakes in the home cages as well as in the observation cages.

We thank the Baltimore City Zoo and Dr. Frank Groves for making facilities available to us. We also thank the M. M.Schmidt Foundation for financial contributions to this work. Reprint requests should be sent to D. Chiszar, Department of Psychology, University of Colorado, Boulder, Colorado 80309.

\section{Procedure}

Permanent postural records of both animals were made by still photography. Two $35-\mathrm{mm}$ cameras were used, one mounted above the cage and another facing the cage front. The cameras were synchronized within $.25 \mathrm{sec}$ so that each posture was photographed simultaneously from the top and from the side. These photographs provided a permanent three-dimensional view of the rattlesnake's postures from which quantitative measures could be taken. Hence, each pair of photographs constituted a single observation. The films were viewed as slides which were projected in pairs. Measurements were taken from these projections and, when necessary, were calculated as a percentage of the image size. This adjusted the measures for any differences which might have existed in their projected sizes as well as for differences in absolute dimensions of the two rattlesnakes. Table 1 presents the labels and definitions of the 27 postural variables.

The snakes were subjected to stimulus conditions which produced various behavioral states: rest, alertness, exploration, predation, and defense. The C. atrox was photographed in all conditions; $S$. miliarus was photographed only in the last two. Rest was produced by a 3-h exposure to nothing other than the ambient stimuli of the room, during which the snake became still. The alert state was produced by exposure to personnel engaged in general activity around the laboratory but not in the immediate vicinity of the cage. Exploration describes a situation following rest when the snake moved about the cage, though without any new or unusual stimulation. Predation occurred when a live mouse was introduced into the cage; the snakes were under 6 days of food deprivation at this time. Defensive behavior was produced by prodding the animals with a snake hook.

The $S$. miliarus was allowed to explore the observation cage for several minutes; it was then disturbed with a metal snake hook. Defense postures were assumed briefly and three pairs of frames were exposed. After $15 \mathrm{~min}$ a mouse was introduced; the snake struck within 1 min. Five pairs of frames were exposed up to and including the strike. Over a period of $3 \mathrm{~min}$, while the snake was waiting for the mouse to die, five more pairs of frames were exposed. Hence, a total of 13 observations of $S$. miliarus were made. The $C$. atrox was left undisturbed for $6.5 \mathrm{~h}$ (during which its behavior ranged from still or resting postures to alert nonactive postures and, finally, to active exploratory postures) 
Table 1

Definitions of 27 Measurements

A. The percent of the total body length that is in physical contact with itself. Because both sides of the snake can be in selfcontact, as in tight coiling, this measurement can be greater than $100 \%$ of the body length. Measured in $C$. attrox only.

A1. The angle between the head and the object (prey or snake hook used to provoke feeding or defense, respectively) such that the snake looking directly at the object forms a 0 -deg angle. Measured in $S$. miliarus only.

B. The angle between the head and neck. If the head is in line with the neck, the angle is 0 deg.

C. The angle between rattle and tail.

D. The percent of the body length seen as overlapping itself when viewed from above. This overlap can occur as in Variable A or when the snake's anterior is elevated over the coils as in defense.

E. The percent of the body length that is used in the formation of body loops.

A body angle was measured as a bend in the body that was bordered by two straight segments. An entire loop was measured as one angle if it was a continuous curve (i.e., $360 \mathrm{deg}$ ). Factors $\mathrm{F}$ through $\mathrm{J}$ represent the number of body angles (in each picture taken by the vertical camera) greater than:
F. 5 deg
G. $45 \mathrm{deg}$
H. $90 \mathrm{deg}$
I. $135 \mathrm{deg}$
J. $\quad 180 \mathrm{deg}$
K. The total number of all the above angles.

L. The number of loops formed by the body. These were measured in C. attrox only.

M. The height to which the head was elevated above the floor, expressed as percent of total body length.

$\mathrm{N}$. The angle between the head and the floor such that a head resting on the floor is 0 deg and an elevated head held parallel to the floor is 0 deg.

O. The height to which the neck was elevated from the floor expressed as percent of total body length.

P. The angle between the neck and floor, following the same criteria as Factor $\mathbf{N}$.

Q. The percent of total body length that is elevated in the anterior region of the body, including the head. Lengths of the body that are resting over another part of the body were considered to be elevated.

R. The height to which the tip of the rattle was elevated above the floor, expressed as percent of body length.

S. The angle between the rattle and the floor following the criteria of Factor $\mathrm{N}$.

T. The height to which the tip of the tail is elevated above the floor, expressed as percent of total body length.

$\mathrm{U}$. The angle between the tail and the floor following the criteria of Factor $\mathrm{N}$.

V. The percent of total body length that is elevated in the posterior region including the rattle.

W. The length of the posture expressed as percent of maximum length (= total body length). Each posture could be encompassed by a polygon when viewed from above. Factor $W$ is the length of the longest diagonal of this polygon, expressed as percent of total body length.

$X$. The length of the smallest diagonal of the above polygon, expressed as percent of total body length.

Y. The percent of the total area (that could be encompassed by the snake in a perfect circle) that is encompassed by the loops of the posture.

Z. The number of tight S-shaped loops seen in the body. These loops were seen only in the anterior region.

and 28 pairs of frames were exposed. The snake was then provoked into a defensive posture; four pairs of frames were exposed. A mouse was introduced $70 \mathrm{~min}$ later. The snake immediately began predatory behavior and three pairs of frames were exposed, during which the mouse was struck. Hence a total of 35 observations of $C$. atrox postures were made.

\section{RESULTS AND DISCUSSION}

Data were subjected to two analyses. First, pictures were grouped according to behavioral states they represented; each variable was then averaged over all pictures within each behavioral state. Composites of variables were made when they measured similar areas of the body (e.g., head and neck) and showed strong covariation. Table 2 presents mean scores for both specimens on each variable (or composite) for each behavioral state. It is clear that behavioral states differed with respect to some of the variables in both specimens. Variables which did not discriminate behavioral states are omitted from Table 2.

The second analysis involved construction of correlation matrices for both specimens: Each variable was correlated with each other variable using the BC-TRY cluster analysis program (Tryon \& Bailey, 1970). Table 3 presents reduced matrices for each specimen; variables which were not correlated with any of the others were omitted from these matrices. The pattern of correlations presented in Table 3 can, of course, be interpreted in a variety of ways. One interpretation centers on the fact that measures of body elevation (anterior and posterior) and attendant angles are fairly well correlated with each other and with the number of S-shaped loops (see correlations contained in the triangles of Table 3). The elevation measures are best detected from the horizontal plane, while the loops are best detected from above. We suggest that this cluster of variables can be taken as a measure of the degree of defensiveness and that the fact of covariation between these variables should not necessarily be understood as a simple redundancy. Indeed, different components of the cluster may well be ritualized as signals for different receivers: The horizontally detectible cues are most appropriate for mustelid or canid predators, while the vertically detectible cues are most appropriate for avian predators or large mammals (e.g., bison) which might accidentally trample the snake if they failed to see or hear it.

It is also noteworthy that most of the behavioral states distinguished in the study are not uniquely as- 
Table 2

Mean Values of Selected Postural Variables or Composites in Each Behavioral State

\begin{tabular}{|c|c|c|c|c|c|c|c|c|c|c|}
\hline \multicolumn{4}{|c|}{ S. miliarus } & \multicolumn{7}{|c|}{ C. atrox } \\
\hline Variable & Defense & Predation & $F(1 / 11)$ & Variable & Defense & Predation & Rest & Alert & Exploration & $F(4 / 30)$ \\
\hline $\begin{array}{l}\text { A1 } \\
\text { FGH } \\
\text { MNOP } \\
\text { RSUV } \\
\text { Z }\end{array}$ & $\begin{array}{l}64 \\
88 \\
79 \\
80 \\
66\end{array}$ & $\begin{array}{l}20 \\
16 \\
44 \\
20 \\
10\end{array}$ & $\begin{array}{c}5.28 * \\
16.85^{* *} \\
8.33^{*} \\
38.97 * * \\
5.20^{*}\end{array}$ & $\begin{array}{l}\text { A } \\
\text { B } \\
\text { C } \\
\text { I } \\
\text { MNOP } \\
\text { SUV } \\
\text { Z } \\
\end{array}$ & $\begin{array}{r}0^{\mathrm{a}} \\
0^{\mathrm{a}} \\
0^{\mathrm{a}} \\
12^{\mathrm{a}} \\
31^{\mathrm{a}} \\
64^{\mathrm{a}} \\
100^{\mathrm{a}} \\
\end{array}$ & $\begin{array}{c}34^{a, b} \\
9^{a} \\
2^{a} \\
83^{b} \\
15^{a, b} \\
38^{a, b} \\
0^{b}\end{array}$ & $\begin{array}{c}80^{\mathrm{b}} \\
55^{\mathrm{b}} \\
45^{\mathrm{b}} \\
50^{\mathrm{a}, \mathrm{b}} \\
0^{\mathrm{b}} \\
0^{\mathrm{b}} \\
0^{\mathrm{b}}\end{array}$ & $\begin{array}{c}47^{\mathrm{a}, \mathrm{b}} \\
18^{\mathrm{a}, \mathrm{b}} \\
10^{\mathrm{a}, \mathrm{b}} \\
28^{\mathrm{a}, \mathrm{b}} \\
16^{\mathrm{a}, \mathrm{b}} \\
28^{\mathrm{a}, \mathrm{b}} \\
8^{\mathrm{b}}\end{array}$ & $\begin{array}{c}0^{\mathrm{a}} \\
0^{\mathrm{a}} \\
0^{\mathrm{a}} \\
50^{\mathrm{a}, \mathrm{b}} \\
83^{\mathrm{c}} \\
55^{\mathrm{a}} \\
0^{\mathrm{b}}\end{array}$ & $\begin{array}{c}4.19^{* *} \\
2.80^{*} \\
2.71^{*} \\
2.29^{\mathrm{M}} \\
11.01^{* *} \\
4.51^{* *} \\
28.55^{* *}\end{array}$ \\
\hline $\mathrm{n}$ & 3 & 10 & & & 4 & 3 & 3 & 23 & 2 & \\
\hline
\end{tabular}

Note-Variables are expressed as mean percentages of maximum values seen under any condition. This was done in order to express all variables in a comparable scale. $M=$ marginally significant $(.10>p>.05) ;{ }^{*} p<.05 ;{ }^{* *} p<.01$; means (within $C$. atrox rows) with common superscripts do not differ significantly by Duncan's Multiple Range test.

Table 3A

Matrix Showing Correlations Between Selected Variables in the Postures of a Specimen of S. miliarus (multiplied by 100)
Table 3B

Matrix Showing Correlations Between Selected Variables in the Postures of a Specimen of C. atrox (multiplied by 100)

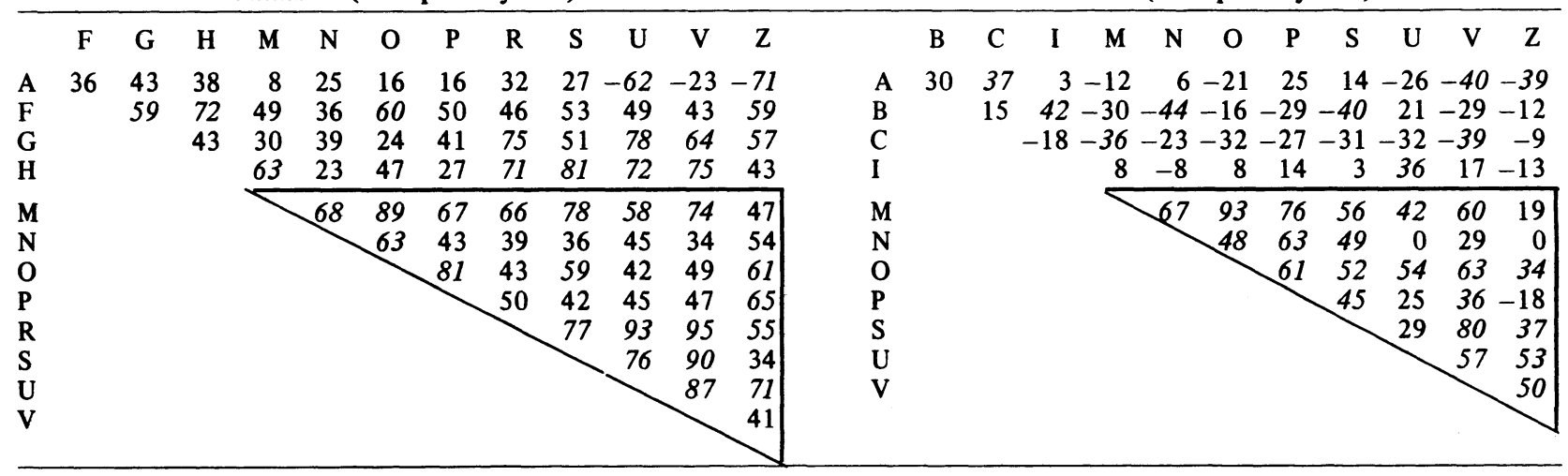

The significant values are in italics $(d f=11, p<.025)$.

The significant values are in italics $(d f=33, p<.025)$.

sociated with specific behavioral display components (Table 2). Whereas quantitative differences between states can be found, qualitative differences generally do not exist. This means that ambiguity is likely to exist from a predator's point of view in that states such as predation, alertness, and exploration may be confused with defensive readiness, especially if cues involving body elevation are utilized. This is obviously advantageous from the rattlesnake's point of view. The fact that the resting state in C. atrox is readily distinguishable from the other states presents no problem because this state is most likely to occur only under protected circumstances or under conditions where shelter is readily accessible. The time when it would be most advantageous (to the snake) for potential predators or other disturbing individuals to receive "erroneous" defensive-readiness cues from a rattlesnake is when the latter is foraging or in transit between resting or hibernating sites.

\section{REFERENCES}

KLAUBER, L. M. Rattlesnakes, their habits, life histories and influence on mankind ( 2 Vols). Berkeley: University of California Press, 1972.

Tryon, R. C., \& BaIley, D. E. Cluster analysis. New York: McGraw-Hill, 1970.

(Received for publication November 29, 1976.) 